\title{
A physically meaningful equivalent circuit network model of a lithium-ion battery accounting for local electrochemical and thermal behaviour, variable double layer capacitance and degradation
}

\author{
Marie-Therese von Srbik*,a, Monica Marinescu ${ }^{b}$, Ricardo F. Martinez-Botas ${ }^{a}$, Gregory J. Offer ${ }^{a}$ \\ ${ }^{a}$ Department of Mechanical Engineering, Imperial College London, UK \\ ${ }^{\mathrm{b}}$ Department of Earth Science and Engineering, Imperial College London, UK \\ *Corresponding author. Department of Mechanical Engineering, Imperial College London, SW7 2AZ United \\ Kingdom. 0049 1731983316, srbik@imperial.ac.uk; Permanent Address: Pienzenauerstreet 17, 81679 \\ Munich, Germany
}

Abstract:

A novel electrical circuit analogy is proposed modelling electrochemical systems under realistic automotive operation conditions. The model is developed for a lithium ion battery and is based on a pseudo 2D electrochemical model. Although cast in the framework familiar to application engineers, the model is essentially an electrochemical battery model: all variables have a direct physical interpretation and there is direct access to all states of the cell via the model variables (concentrations, potentials) for monitoring and control systems design. This is the first Equivalent Circuit Network -type model that tracks directly the evolution of species inside the cell. It accounts for complex electrochemical phenomena that are usually omitted in online battery performance predictors such as variable double layer capacitance, the full current-overpotential relation and overpotentials due to mass transport limitations. The coupled electrochemical and thermal model accounts for capacity fade via a loss in active species and for power fade via an increase in resistive solid electrolyte passivation layers at both electrodes. The model's capability to simulate cell behaviour under dynamic events is validated against test procedures, such as standard battery testing load cycles for current rates up to $20 \mathrm{C}$, as well as realistic automotive drive cycle loads. 
1. Introduction

It is critical for the commercial success of modern electronic products as well as electric vehicles to provide the user with accurate battery range estimation based on in-situ battery state predictions. Important parameters for arriving at an accurate range prediction are State of Charge (SOC) and State of Health $(\mathrm{SOH})$. Equivalent circuit network (ECN) models are particularly important to control engineers as a tool to monitor in-use performance through battery management systems. Whilst efficient in computing battery cell voltage, they do not distinguish the underlying causes of degradation or variations in performance [16]. For accurate in-situ SOC and $\mathrm{SOH}$ predictions and monitoring more information than overall cell voltage is required [7]. The Voltage-SOC charge/discharge characteristics are progressively changing as a consequence of complex interrelated phenomena and degradation processes [8]. This fact gives rise to the need for a model able to predict the relevant electrochemical, mass transport and thermal processes.

In the model presented here, the cell voltage response to a load emerges as the coupled effect of individual electrochemical and thermal processes, translated into electrical analogues. As such, the electrochemical and thermal phenomena in the system are 'transparent' in this forward facing battery model, as parameters can be evaluated without the need for iterative backwards evaluation steps. Every electrical element retains a distinct physical meaning in terms of potentials, degradation mechanisms and heat generation. System control algorithms can be implemented accordingly to monitor the evolution of these parameters.

In-situ models commonly comprise of Voltage-SOC look-up algorithms and discharge curve interpolation, as proposed by Tremblay [9]. Whilst being fast, these have the significant drawback that their validity is 
limited to the specific operating conditions under which the look-up table was generated $[10,11]$. It is experimentally extremely costly to populate voltage look-up tables for a comprehensive range of operating conditions, including current, temperature and cycle number, in all the relevant combinations. The most advanced developments of phenomenological ECN models presented by Bergveld [12] and Raël et al. [13] fail to account for a full thermal discretisation and mass transport, limiting them to low C-rate applications. They also do not account for variable double layers, limiting them to low frequency applications, or for variable passivating layers, making them unsuitable for predicting degradation.

\section{Phenomenological battery model}

The 1D lithium ion cell model presented in this paper consists of three domains: a positive (metal oxide) electrode, an electronically insulating but ionically conducting separator and a negative (carbon) electrode. Both electrodes in the system are electronic conductors, each with an electrical potential $\phi$, due to the electrochemical potential $\bar{\mu}$ of lithium stored in them.

As a design feature, the chemical potential $\mu$ of the anode is always larger than that of the cathode. The resulting difference in chemical potentials between the electrodes acts as the driving force for electrochemical reactions during operation. Throughout discharge (Fig. 1), the cathode chemical potential is always below that of the anode. The anode releases lithium-ions $\mathrm{Li}^{+}$into the electrolyte, thereby increasing its electrical potential $\phi^{\mathrm{a}}$ towards equilibrium with respect to $\mathrm{Li} / \mathrm{Li}^{+}$. These lithium-ions move towards the more favourable thermodynamic state, decreasing the cathode electrical potential $\phi^{\mathrm{c}}$. The resulting ionic flux from anode to cathode occurs simultaneously to an electron (e-) flow in the external circuit. 
The model thus accounts for three species (lithium-ions in the electrolyte, electrons in binders and current collectors, and lithium intercalated in the active electrode material) and two modelling domains (chemical inside electrodes and electrical in electrolyte and external circuit). The system is modelled according to porous electrode theory, with solid and electrolyte phases being treated as superimposed homogeneous continua.

The three networks, one for each of the three species present, electrons, $\mathrm{Li}^{+}$, and intercalated lithium, are connected through a triple species element (TSE), which governs the conversion between species taking into account losses in the form of activation, diffusion and passivating layer overpotentials, as discussed below. The TSE is an essential feature of this model, coupling information from each of the three joining circuit networks.

The current-overpotential relation implemented for each electrode [14] is given by

$i_{\mathrm{t}}=\left(i_{\mathrm{Red}}-i_{\mathrm{Ox}}\right)=i^{0} a_{\mathrm{s}}\left[\left(\frac{c_{\mathrm{Red}}^{\mathrm{s}}}{c_{\mathrm{Red}}^{\mathrm{b}}}\right) \exp \left(\frac{\alpha^{\mathrm{a} R}}{\mathrm{RT}} \eta^{\mathrm{ct}}\right)-\left(\frac{\mathrm{c}_{\mathrm{Ox}}^{\mathrm{s}}}{c_{\mathrm{OX}}^{\mathrm{b}}}\right) \exp \left(\frac{\alpha^{\mathrm{c}} \mathrm{R}}{\mathrm{RT}} \eta^{\mathrm{ct}}\right)\right]$.

It can be seen that Eq. (1) is a function of bulk and surface species concentrations of the oxidised and reduced species. The assumption that reduction/oxidation surface and bulk species concentrations are equal at all times $\left(c_{\mathrm{Red}}^{\mathrm{s}}=c_{\mathrm{Red}}^{\mathrm{b}}\right.$ and $\mathrm{c}_{\mathrm{Ox}}^{\mathrm{s}}=\mathrm{c}_{\mathrm{Ox}}^{\mathrm{b}}$ ) leads to a simplification of Eq. (1); the Butler-Volmer (BV) equation [15]. This simplification is made in many Li-ion battery models $[13,16-20]$ and implies that there are no mass transport limitations in the electrolyte, and therefore no diffusion overpotentials as part of the charge-transfer overpotentials. This simplification is only valid for relatively low currents, and will thus not be made in this model. The charge-transfer overpotential $\eta^{\text {ct }}$ in Eq. (1) is therefore composed not only of the activation overpotential $\eta^{\text {act }}$ (as in the BV- equation), but also of the diffusion overpotentials due to 
the transport of lithium in each electrode $\eta_{\mathrm{Li} i}^{\mathrm{d}}$ and lithium ion in the electrolyte $\eta_{\mathrm{Li}^{+}}^{\mathrm{d}}$, and of the overpotential due to the passivating layer $\eta^{\mathrm{pl}}$ at either electrode:

$\eta^{\mathrm{ct}}=\eta_{\mathrm{Lii}}^{\mathrm{d}}+\eta_{\mathrm{Li}^{+}}^{\mathrm{d}}+\eta^{\mathrm{act}}+\eta^{\mathrm{pl}}$

The exchange current density in Eq. (1) denotes the reaction current flowing at zero overpotential $[17,21]$ and can be expressed as

$i^{0}=n \mathrm{~F} a_{\mathrm{s}}\left(k_{\mathrm{a}}\right)^{\alpha^{\mathrm{a}}}\left(k_{\mathrm{c}}\right)^{\alpha^{\mathrm{c}}}\left(c_{i}^{\mathrm{b}}\right)^{\alpha^{\mathrm{c}}}\left(c_{\mathrm{Li} i}^{\mathrm{b}}\right)^{\alpha^{\mathrm{a}}}$

The potential difference at the electrode/electrolyte interface can be expressed as

$\left(\phi^{\mathrm{s}}-\phi^{\mathrm{l}}\right)=E^{\mathrm{eq}}+\eta_{\mathrm{Li} i}^{\mathrm{d}}+\eta_{\mathrm{Li}^{+}}^{\mathrm{d}}+\eta^{\mathrm{act}}+\eta^{\mathrm{pl}}$

Eq. (4) can be interpreted with the help of electric circuit elements. Fig. 2 shows the constituent electrode potential losses (top) included in the proposed model as well as the introduction of the TSE (bottom). The electrode equilibrium potential (itself a function of the material Nernst potential for the intercalation reaction [14]) is reduced by all present overpotentials that act as losses. A capacitor is placed in parallel with the TSE to represent the double-layer capacitance present at the electrode surface, discussed in Section 2.4.

\subsection{Diffusion overpotentials}

Due to the finite velocity of diffusion for $\mathrm{Li}^{+}$and intercalated $\mathrm{Li}$, concentration gradients occur inside electrodes and electrolyte, especially at high currents. The bulk species properties temporarily deviate 
from those at the surface, where the intercalation process occurs. This local concentration variation causes a potential drop, represented by diffusion overpotentials.

The concentration gradients occurring inside an electrode during lithiation and de-lithiation have been related to electrical and elastic interaction forces between the $\mathrm{Li}^{+}$ions and the host atoms leading to phase changes [22]. As a result, the contributions to diffusion overpotential can be expressed as $\eta_{\mathrm{Li} i}^{\mathrm{d}}$, the overpotential due to intercalated lithium diffusion to the reaction site

$\eta_{\mathrm{Li} i}^{\mathrm{d}}=\frac{\mathrm{R} T}{n \mathrm{~F}} \ln \left(\frac{c_{i}^{\mathrm{s}} c_{\mathrm{Li} i}^{\mathrm{b}}}{c_{\mathrm{Li} i}^{\mathrm{s}} c_{i}^{\mathrm{b}}}\right)$

and $\eta_{\mathrm{Li}^{+}}^{\mathrm{d}}$, the overpotential at the interface due to diffusion of lithium-ions in the vicinity of the reaction site:

$\eta_{\mathrm{Li}^{+}}^{\mathrm{d}}=\frac{\mathrm{R} T}{n \mathrm{~F}} \ln \left(\frac{c_{\mathrm{Li}^{+}}^{\mathrm{s}}}{c_{\mathrm{Li}^{+}}^{\mathrm{eq}}}\right)$

to be substituted in Eq. (4).

\subsection{Activation overpotentials}

The activation overpotential required for an electrochemical reaction to occur is related to the change in Gibbs free energy during the reaction. It is only present when an electrochemical reaction takes place. In Eq. (4) the activation overpotential $\eta^{\text {act }}$ for each electrode is substituted by the expression derived by Prada et al. [23]: 


$$
\eta^{\text {act }}=\frac{\mathrm{R} T}{\alpha n \mathrm{~F}} \ln \left(\xi+\sqrt{\xi^{2}+1}\right), \text { where } \quad \xi=\frac{r_{\mathrm{s}}}{6 \varepsilon_{\mathrm{s}} i^{0} a_{\mathrm{s}} \delta} I
$$

\subsection{Passivating layer overpotentials}

A passivating layer, also on the anode sometimes referred to as the solid electrolyte interphase layer (SEI) can form at the electrode/electrolyte interface due to the presence of parasitic side-reactions. In order to account for its effects on battery performance, a layer of thickness $\delta^{\mathrm{pl}}$ is considered to physically separate reactants and products, acting as a resistance against the charge-transfer process. As a measure of cell degradation with cycling, the layer thickness and resistance are considered to increase at a rate depending on the local current density and temperature. Also as a result of these parasitic reactions, active lithium is irreversibly deposited at the layer, such that it no longer participates in the reaction process, leading to capacity fade. The presence of a passivating layer also introduces an effective resistance $R^{\mathrm{pl}}$, contributing to an increase in cell internal impedance, and thus causing power fade. Prada et al.'s approach is adopted in describing the effects of this layer [24]. The passivation layer overpotential is the voltage drop across the layer:

$\eta^{\mathrm{pl}}=-\frac{i_{\mathrm{t}} R^{\mathrm{pl}}}{a_{\mathrm{s}}}$

while the rate of increase in its resistance is

$\frac{\mathrm{d}}{\mathrm{d} t} R^{\mathrm{pl}}=\frac{1}{\kappa^{\mathrm{pl}} S} \frac{\mathrm{d}}{\mathrm{d} t} \delta^{\mathrm{pl}} \quad$, where $\quad S=3 \varepsilon_{\mathrm{s}} \delta^{\mathrm{pl}} \frac{a_{\mathrm{s}}}{r_{\mathrm{s}}}$

and the rate of increase in its thickness is 
$\frac{\mathrm{d}}{\mathrm{d} t} \delta^{\mathrm{pl}}=-\frac{i^{\mathrm{pl}}}{2 \mathrm{~F}} \frac{M^{\mathrm{pl}}}{\rho^{\mathrm{pl}}}$

Besides causing capacity and power fade, the process of layer growth also reduces the useful local intercalation current density:

$i_{\mathrm{t}}=i^{0}+i^{\mathrm{pl}} \quad$,

where the total current density $i_{\mathrm{t}}$ comprises, the useful intercalation current density $i^{0}$, and the sidereaction current density $i^{\mathrm{pl}}$. The side-reaction current density may be formulated according to the Tafel equation [14], under the assumption that the reverse reaction contributes less than $1 \%$ to the exchange current density, i.e. the main contribution to the growth of the layer comes from an irreversible reaction [25]:

$i^{\mathrm{pl}}=-i^{0} a_{\mathrm{s}} \exp \left(\frac{\mathrm{nF}}{2 \mathrm{R} T} \eta^{\mathrm{pl}}\right)$

The total capacity loss incurred by time $t$ due to the loss of active material from growing the passivation layer at one electrode is calculated as a function of the side reaction current:

$Q^{\mathrm{pl}}(t)=-\int_{0}^{t} \int_{0}^{\mathrm{L}} i^{\mathrm{pl}} \mathrm{d} x \mathrm{~d} t$

\subsection{Electrochemical double layer}

The electrochemical charge-transfer process takes place at the interface between the charged electrodes and the electrolyte. This region is not only occupied by any passivation layer, but also populated by an 
electrochemical double-layer. This layer is formed by the mobile ions in the electrolyte, attracted to screen the electrode potential. The high ionic concentrations inherent to the double layer are expected to affect ionic transport to and from the interface, and therefore affect the intercalation rate.

According to the Gouy-Chapman theory, the distance over which the potential drop $\eta^{\mathrm{pl}}$ due to the presence of the double layer occurs, can be characterised by the Debye length $\lambda$ [26]. The Debye length, a measure of the effectiveness of screening, increases with decreasing bulk lithium-ion concentration $c_{\mathrm{Li}^{+}}$:

$$
\lambda=\sqrt{\frac{\mathrm{R} T \varepsilon_{0} \varepsilon_{\mathrm{r}}^{\mathrm{dl}}}{2 \mathrm{~F}^{2} c_{\mathrm{Li}^{+}}}}
$$

Oldham et al. modified the expressions first proposed by Kornyshev [27] for electrode/electrolyte interfaces, by relating the electrical potential difference between the electrode/electrolyte interface $\phi^{\mathrm{l}}(0)$ and the bulk electrolyte $\phi^{\mathrm{l}}(\infty)$ to the charge density $q$ on the interface.

The potential drop across the double layer comprises of an inner layer potential drop (Stern layer), which is a function of the size of a lithium-ion $r_{\mathrm{Li}^{+}}$, and of an outer Debye layer potential drop. As the two layers are electrically in series, the overall capacitance density of the interface can be expressed as

$$
\frac{1}{C^{\mathrm{dl}}}=\frac{\phi^{\mathrm{l}}(0)-\phi^{\mathrm{l}}(\infty)}{q}=\frac{1}{C_{\mathrm{inner}}}+\frac{1}{C_{\text {outer }}}
$$

where

$$
C_{\text {inner }}=\frac{\varepsilon_{0} \varepsilon_{\mathrm{r}}^{\mathrm{dl}}}{r_{\mathrm{Li}^{+}}} \quad \text { and }
$$


$C_{\text {outer }}=\frac{\sqrt{\varepsilon_{0} \varepsilon_{\mathrm{r}}^{\mathrm{dl}}}}{\lambda} \frac{q}{\sqrt{8 \mathrm{R} T c_{\mathrm{Li}^{+}}(x)} \arcsin \left\{\frac{q}{8 \mathrm{R} T c_{\mathrm{Li}^{+}}(x)}\right\}}$

Only the outer layer capacitance varies as a function of lithium-ion concentration variation, while the inner layer capacitance is linear with its thickness. Formulated in this way, local exchange current density is a function of the arrangement of the two layers and since it is itself a function of active electrode surface area implicitly reflects the pseudocapacitance of the electrode electrolyte interphase. Pseudocapacitance due to interaction of solvated ions and the electrode is an important aspect in modelling electrochemical processes of nanostructured electrodes and is often omitted in battery models. [28-31]

The overpotential due to the charged double layer capacitance can be expressed as

$\eta^{\mathrm{pl}}(t)=\int i^{0} d t C^{\mathrm{dl}}(t)$

2.5. Species transport in the electrodes and electrolyte

The transport of species inside electrodes and through the electrolyte is modelled as in Fuller, Doyle and Newman [32]. Diffusion of $\mathrm{Li}^{+}$in the electrolyte is governed by Fick's second law

$\frac{\partial\left(\varepsilon_{1} c_{\mathrm{Li}^{+}}\right)}{\partial t}=\frac{\partial}{\partial x}\left(D_{\mathrm{eff}} \frac{\partial c_{\mathrm{Li}^{+}}}{\partial x}\right)+\frac{1-t_{+}^{0}}{F} i_{\mathrm{t}} \quad$,

with the boundary conditions at the current collector accounting for the fact that no ions can be present in the current collectors:

$\left.\frac{\partial\left(c_{\mathrm{Li}^{+}}\right)}{\partial x}\right|_{x=0}=\left.\frac{\partial\left(c_{\mathrm{Li}^{+}}\right)}{\partial x}\right|_{x=\delta_{+}}=0$ 
For intercalated lithium transport by diffusion inside the electrodes, Fick's second law is applied in spherical coordinates, resulting in

$\frac{\partial c_{\mathrm{Li} i}}{\partial t}=\frac{\partial D_{\mathrm{Li} i}}{r^{2}} \frac{\partial}{\partial r}\left(r^{2} \frac{\partial c_{\mathrm{Li} i}}{\partial r}\right)$

with boundary conditions enforcing a zero gradient concentration at the centre of the particle (due to symmetry) and the conservation of charge between the solid and liquid phase at the particle boundary:

$\left.\frac{\partial\left(c_{\mathrm{Li} i}\right)}{\partial r}\right|_{r=0}=0$ and $-\left.D_{\mathrm{s}} \frac{\partial\left(c_{\mathrm{Li} i}\right)}{\partial r}\right|_{r=r_{\mathrm{s}}}=\frac{i_{\mathrm{t}}}{a_{\mathrm{s}} \mathrm{F}}$.

2.6. Electrolyte potential

Charge conservation in the electrolyte phase is maintained according to $[17,18,33]$

$\frac{\partial}{\partial x}\left(\kappa^{\mathrm{eff}} \frac{\partial \phi^{\mathrm{l}}}{\partial x}\right)+\frac{\partial}{\partial x}\left(\kappa_{\mathrm{D}}^{\mathrm{eff}} \frac{\partial}{\partial x} \ln c_{\mathrm{Li}^{+}}\right)+i_{\mathrm{t}}=0 \quad$,

which relates the electrical potential of the electrolyte with the ion concentration and the reaction current density. A zero potential gradient is applied at each current collector interface, reflecting that charge cannot accumulate on the current collector.

$\left.\frac{\partial \phi^{\mathrm{l}}}{\partial x}\right|_{x=0}=\left.\frac{\partial \phi^{\mathrm{l}}}{\partial x}\right|_{x=\delta_{+}}=0$ 
Inside the electrodes, Ohm's law accounts for charge conservation according to

$\frac{\partial}{\partial x}\left(\sigma^{\mathrm{eff}} \frac{\partial \phi^{\mathrm{s}}}{\partial x}\right)=i_{\mathrm{t}}$

As for the electrode/current collector interface, there is no charge accumulation at the electrode/separator interface:

$\left.\frac{\partial \phi^{\mathrm{s}}}{\partial x}\right|_{x=\delta_{-}}=\left.\frac{\partial \phi^{\mathrm{s}}}{\partial x}\right|_{x=\delta_{+}}=0$

The electrical current obtained in the outside circuit results from the potential gradient at the electrode/current collector interface.

$-\left.\sigma_{\text {neg }}^{\text {eff }} \frac{\partial \phi^{\mathrm{s}}}{\partial x}\right|_{x=0}=\left.\sigma_{\text {pos }}^{\text {eff }} \frac{\partial \phi^{\mathrm{s}}}{\partial x}\right|_{x=\delta_{+}}=\frac{I_{\mathrm{bat}}}{A_{\mathrm{s}}}$.

\subsection{Electric circuit elements}

The transport phenomena of lithium-ions in electrolyte and intercalated lithium in electrodes are translated into phenomenological electric circuit elements, as detailed below. These elements form the building blocks of the sub-circuits governing the transport of the individual species, which are assembled into a complete circuit network, connected by elements governing the electrochemical reactions in the system.

As lithium-ions are charged species, they are treated in a similar manner as electrons, such that the circuit elements governing the transport of both these species reflect electrical quantities, in the electrical 
domain, in units of $[\mathrm{V}],[\mathrm{A}],[\Omega]$ and $[\mathrm{F}]$. Intercalated lithium is uncharged, such that the circuit elements governing its transport reflect equivalent chemical quantities, in the chemical domain, in units of [J mol $\left.{ }^{-1}\right]$, $\left[\mathrm{mol} \mathrm{s}^{-1}\right],\left[\mathrm{J} \mathrm{s} \mathrm{mol}^{-2}\right]$ and $\left[\mathrm{mol}^{2} \mathrm{~J}^{-1}\right]$.

The electrode interface potentials $\phi[\mathrm{V}]$ are linked by the TSE to the corresponding chemical potentials $\mu$ [ $\mathrm{mol}^{-1}$ ] by a factor of $1 / n \mathrm{~F}$, where $n$ is the number of electrons participating in the reaction. The TSE therefore represents the translation node where chemical and electrical quantities are linked. The unit conversion can be verified by the unit analysis in Eq. (27):

$\phi=\frac{\mu}{n \mathrm{~F}} \equiv\left[\frac{\mathrm{J}}{\mathrm{mol}}\right]\left[\frac{\mathrm{C}}{\mathrm{mol}}\right]^{-1} \equiv\left[\frac{\mathrm{J}}{\mathrm{C}}\right] \equiv[\mathrm{V}]$

With this same conversion factor one can retrieve further chemical equivalents to electrical quantities, such as the relation between electron flux $I$ (i.e. current) in units of $[\mathrm{A}]$ and ionic flux $J^{\text {ch }}$ (i.e. chemical species flux) in the units of $\left[\mathrm{mol} \mathrm{s}^{-1}\right]$ :

$I=n \mathrm{~F} J^{\mathrm{ch}} \equiv\left[\frac{\mathrm{C}}{\mathrm{mol}}\right]\left[\frac{\mathrm{mol}}{\mathrm{s}}\right] \equiv\left[\frac{\mathrm{C}}{\mathrm{s}}\right] \equiv[\mathrm{A}]$

A capacitor is the element of choice for simulating the effect of local species accumulation manifesting itself in concentration gradients, while resistors are chosen to reflect resistance to species transport. The expressions for the resistors and capacitors in each domain are derived below.

In the chemical domain the charge on the capacitor corresponds to the concentration of species in a domain. The domain's electrochemical potential $\bar{\mu}_{i}$ can be expressed as the sum of its constituents: the standard redox potential $\mu_{i}^{0}$, the concentration potential which can also include diffusion limitations 
effects $\mathrm{R} T \ln \left(\frac{c_{i}}{c_{i}^{\text {ref }}}\right)$ and the electrostatic potential due to an external electric field, such as that of the charged electrode, acting on the species, $z_{i} \mathrm{~F} \phi_{i}$ :

$\bar{\mu}_{i}=\mu_{i}^{0}+\mathrm{R} T \ln \left(\frac{c_{i}}{c_{i}^{\mathrm{ref}}}\right)+z_{i} \mathrm{~F} \phi_{i}$

where $c_{i}$ denotes the concentration of a species.

The expression for the chemical capacitance (in $\left[\mathrm{mol}^{2} \mathrm{~J}^{-1}\right]$ ) of the capacitor with potential as given by Eq. (29) can be derived by analogy to a dielectric capacitor.

$\frac{1}{C^{\mathrm{ch}}(c)}=\Delta x \frac{\partial \bar{\mu}_{i}}{\partial c}=\Delta x \frac{\partial}{\partial c_{i}}\left(\mu_{i}^{0}+\mathrm{R} T \ln \left(\frac{c_{i}}{c_{i}^{\mathrm{ref}}}\right)\right)=\Delta x \frac{\mathrm{R} T}{c_{i}}$

This relation can be applied to the chemical capacitance corresponding to the intercalated lithium inside the electrodes. As this domain is modelled as spherical particles, Eq. (30) is expressed in spherical coordinates. The chemical capacitance of a spherical shell volume at distance $r_{j}$ from the particle centre is:

$C_{\mathrm{Li} i}^{\mathrm{ch}}\left(r_{j}\right)=\frac{c_{\mathrm{Li} i}\left(r_{j}\right)}{\mathrm{R} T\left(r_{j}\right)} \cdot \frac{4}{3} \pi\left(r_{j}-r_{j-1}\right)^{3}$

Using Eq. (31) and the $1 / n \mathrm{~F}$ conversion factor, the expression for the electrical capacitance in [F] of any discretised electrolyte volume due to the presence of $\mathrm{Li}^{+}$can be derived in the electrical domain. This domain is modelled as rectangular prisms and is therefore expressed in Cartesian coordinates: 
$C_{\mathrm{Li}^{+}}^{\mathrm{el}}\left(x_{j}\right)=\frac{C_{\mathrm{Li}^{+}}\left(r_{j}\right)}{\mathrm{RT}\left(x_{j}\right)(n \mathrm{~F})^{2}} \cdot A_{\mathrm{s}}\left(x_{j}-x_{j-1}\right)$

Mass transport is modelled by a resistor-capacitor (RC) arrangement, shown in Fig. 3 in the electrolyte (in Cartesian coordinates) for the electrical domain and in the electrode (in spherical coordinates) for the chemical domain.

The local variation of species concentration in the electrodes leads to species fluxes across the interfaces of the control volumes. Transport from a region with high electrochemical potential to one with low electrochemical potential can be expressed as a flux of species $i$ [34]:

$J_{i}^{\mathrm{ch}}\left(r_{j}\right)=-\frac{D_{\mathrm{i}} c_{i}\left(r_{j}\right)}{\mathrm{RT}\left(r_{j}\right)} \frac{\partial \bar{\mu}_{i}}{\partial r} \cdot\left(r_{j}-r_{j-1}\right)$

From the expressions of capacitance and flux an expression for resistance can be obtained using Ohm's law. The species flux caused by the electrochemical potential gradient must overcome the resistivity of the medium, here modelled by a chemical resistance. In general for a species $i$ :

$J_{i}^{\mathrm{ch}}\left(r_{j}\right)=-\frac{1}{R_{i}^{\mathrm{ch}}\left(r_{j}\right)} \cdot \frac{\partial \bar{\mu}_{i}}{\partial r}$

Substitution of Eq. (33) into (34) allows the definition of the resistance $R_{\mathrm{Lii}}^{\mathrm{ch}}\left(r_{j}\right)$ in [J s mol${ }^{-2}$ ] to the lithium flux in the electrodes (chemical domain):

$R_{\mathrm{Li} i}^{\mathrm{ch}}\left(r_{j}\right)=\frac{\mathrm{R} T\left(r_{j}\right)}{c_{\mathrm{Li} i}\left(r_{j}\right) D_{\mathrm{Li} i}} \cdot\left(r_{j}-r_{j-1}\right)^{-1}$ 
Analogous to the methodology applied for deriving chemical capacitances, the $1 / n \mathrm{~F}$ conversion factor is used to obtain the expression for the resistance in $[\Omega]$ to a flux of lithium-ions through electrolyte in Cartesian coordinates (electrical domain):

$R_{\mathrm{Li}^{+}}^{\mathrm{el}}\left(x_{j}\right)=\frac{\mathrm{R} T\left(x_{j}\right)}{c_{\mathrm{Li}^{+}}\left(x_{j}\right) D_{\mathrm{Li}^{+}}(n \mathrm{~F})^{2}} \cdot\left(x_{j}-x_{j-1}\right)^{-1}$.

The expressions for $R^{\mathrm{el}}$ and $C^{\mathrm{el}}$ allow the calculation of the parallel RC characteristic response time as $\Delta x^{2} / D$, which is in accordance with the derivations by Horno et al. [35]. The corresponding energy and power expressions can be derived as summarised in Tab. 1.

The equivalent circuits for the three sub-systems (intercalated lithium, lithium-ions and electrons) are combined, as shown in Fig. 3. As can be seen the TSE links the e circuit containing an electron transport resistance $R_{\mathrm{e}^{-}}^{\mathrm{e}}$, the intercalated lithium circuits in the electrodes and the lithium-ion circuit in the electrolyte. The translation of the particle-structure electrode into a single particle system and subsequently into an electrical network analogy is shown. The un-charged intercalated lithium is shown inside the particle, arranged below the TSE in the Fig. 3, while the charged lithium-ions and electrons are shown in the electrolyte and electron conducting circuit, arranged above the TSE in the Figure.

\subsection{Thermal model}

The electrochemical and physical processes inside the cell cause reversible and irreversible heat generation, leading to temperature variations during operation. The local temperature in the cell, in turn, affects material properties and reaction rates. The temperature dependence of reaction rates is commonly assumed to follow an Arrhenius relation: 
$\psi=\psi_{\text {ref }} \exp \left[\frac{E_{\psi}^{\text {act }}}{\mathrm{R}}\left(\frac{1}{T_{\text {ref }}}-\frac{1}{T}\right)\right]$

The temperature dependency of the following parameters and functions is modelled according to Eq. (37): exchange current densities $i^{0}$, diffusion coefficients in the electrolyte $D_{\mathrm{Li}^{+}}$and in the electrodes $D_{\mathrm{Li} i}$, ionic conductivities $\kappa_{\mathrm{Li} i}$ and double layer capacitances $C_{i}^{\mathrm{dl}}$.

The thermal model employed follows the method presented by Smith and Wang [17]. The heat generation or absorption in a cell during operation is due to a combination of reaction heat $\left(q_{\mathrm{r}}\right)$, ohmic heat from lithium transport through the electrode and electrolyte phases $\left(q_{\mathrm{j}}\right)$, ohmic heat due to the contact resistance between the electrodes and current collectors (electron transport) $\left(q_{\mathrm{c}}\right)$ and entropic heat due to phase changes of the electrode material during intercalation/de-intercalation $\left(q_{\mathrm{e}}\right)$.

Conservation of thermal energy in the system therefore dictates a balance between local heat generation/absorption terms and heat accumulation and convective dissipation at every location, given the local material properties:

$\rho C_{\mathrm{p}} \frac{\partial T}{\partial t}=\frac{\partial}{\partial t}\left(k_{\mathrm{t}} \frac{\partial T}{\partial x}\right)+\left(q_{\mathrm{r}}+q_{\mathrm{j}}+q_{\mathrm{c}}+q_{\mathrm{e}}\right)$

with boundary conditions reflecting free air convective heat dissipating away from the battery surface (which is in this case just one electrode pair of anode, cathode and separator).

$\left.\frac{\partial T}{\partial x}\right|_{x=0}=\left.\frac{\partial T}{\partial x}\right|_{x=L}=\frac{h}{k} A_{\mathrm{s}}\left(T-T_{\mathrm{amb}}\right)$. 


$$
\begin{aligned}
& q_{\mathrm{r}}=A_{\mathrm{s}} \int_{x}^{x+d x} i_{\mathrm{t}}\left(\phi^{\mathrm{s}}-\phi^{\mathrm{l}}-E^{\mathrm{eq}}\right) \mathrm{d} x \\
& q_{\mathrm{e}}=a_{s} i_{t} T \frac{\partial E^{\mathrm{eq}}}{\partial T}, \\
& q_{\mathrm{j}}=A_{\mathrm{s}} \int_{x}^{x+d x}\left[\sigma_{\mathrm{Li} i}^{\mathrm{eff}}\left(\frac{\partial \phi^{\mathrm{s}}}{\partial x}\right)^{2}+\kappa_{\mathrm{Li}^{+}}^{\mathrm{eff}}\left(\frac{\partial \phi^{\mathrm{l}}}{\partial x}\right)^{2}+\kappa_{\mathrm{D}}^{\mathrm{eff}}\left(\frac{\partial \ln c_{\mathrm{Li}^{+}}}{\partial x}\right)\left(\frac{\partial \phi^{\mathrm{l}}}{\partial x}\right)\right] \mathrm{d} x \\
& q_{\mathrm{c}}=\left(I_{\mathrm{bat}}\right)^{2} \frac{R_{\mathrm{c}}}{L_{\mathrm{x}} L_{\mathrm{z}}} .
\end{aligned}
$$

\subsection{Model assembly}

The modelling unit consisting of an electrode pair, separator and current collectors is shown in Fig. 4. This illustrative implementation consists of three discretisation particles in each electrode and three discretisation elements in the separator. The number of discretisation units can be modified; the depicted discretisation was deemed sufficient to provide results to a high enough accuracy for the current rates tested. Fig. 4 illustrates the model derivation, from the schematic of a particle-like two-dimensional electrode pair (top), through the pseudo-2D Newman model type approximation (middle) and finally to its representation as an electrical network analogy (bottom). The direction of current flow is indicated during discharge. In this case lithium de-intercalates from the negative electrode, as indicated by the chemical flux arrow and intercalates into the cathode, again shown by the flux arrow into the particles of the positive electrode.

Circuit layout: In Fig. 4 the cathode is shown on the right hand side of the model, the anode is shown on the left hand side with the separator region in-between. By discretising the electrodes in the x-direction, the model allows the electrode region (particle) closest to the separator to be charged/discharged first, 
followed by the particles further away. The electrolyte domain is discretised at each particle location and throughout the separator. Concentration gradients, and consequently gradients of all other electrochemical and thermal properties in the electrolyte, build across the electrode (and separator) thickness. This effect becomes essential for high C-rate and highly dynamic operations, when mass transport limitations cannot be ignored, and is not accounted for in previously reported phenomenological equivalent circuit battery models $[7,12,13]$.

Modelling domains: The sub-circuits governing the transport of uncharged species i.e. intercalated lithium in the electrodes, are included in the chemical modelling domain. Inside the electrodes, resistances oppose the lithium flow from one particle shell to the next and to the electrode/electrolyte interface where the reaction takes place. The amount of lithium contained in each shell is represented by its capacitance. The relevant chemical quantities are the chemical potential in $\left[\mathrm{J} \mathrm{mol}^{-1}\right]$, chemical flux in $\left[\mathrm{mol} \mathrm{sec}^{-1}\right]$, chemical resistance in $\left[\mathrm{J} \mathrm{mol}^{-2}\right]$ and chemical capacitance in $\left[\mathrm{mol}^{2} \mathrm{~J}^{-1}\right]$.

The sub-circuits governing the transport of charged species i.e. (negative) electrons and (positive) lithiumions are modelled in the electrical modelling domains. The resistance to lithium-ion flux in the electrolyte from one electrode to the other through the separator is modelled by resistors, while the amount of ions stored in each discretised volume is represented by a capacitor. The relevant electrical quantities are the electrical potential in $[\mathrm{V}]$, electrical flux (current) in $[A]$, electrical resistance in $[\Omega]$ and electrical capacitance in $[\mathrm{F}]$. The chemical and the electrical domains are linked by a conversion factor of $1 / n F$, where $n$ is the number of electrons participating in each reaction, here equal to unity.

Special model features: Local electrode surface passivating layers form and grow during operation due to parasitic side-reactions taking place in parallel to the desired reactions. The build-up of this layer leads to lithium-ions being tied up locally and no longer participating in the redox shuttle; manifesting itself in the 
form of capacity fade. Furthermore, any increase in the passivating layer thickness leads to an increase in internal impedance, also observable as power fade.

Triple Species Elements link the chemical sub-circuits and the electrical sub-circuits. At the electrode/electrolyte interface the local electrode potential and chemical species flux are coupled to the electrolyte potential and charged species fluxes. This link is established by a conversion factor of $1 / n F$. The top edge of each TSE therefore represents the electrical potential of the of the charged species present at the interface, such as electrons and $\mathrm{Li}^{+}$, while the bottom corner represents the local chemical potential of the intercalated lithium. As indicated by the schematic of the TSE, each corner is figuratively connected to one of the three species linked by it. While the left-hand side is connected to the electron transport circuit, the right-hand side is connected to the electrolyte transport system and the bottom corner is connected to the transport network inside the electrode.

A capacitor representing the double-layer capacitance at the electrode surface is arranged in parallel with each TSE element and varies with electrochemistry and temperature. This allows the prediction of local ionic concentration gradients that affect ionic transport to and from the interface and therefore impacts on the electrochemical reaction rate and thus on the dynamic cell behaviour.

As described by Eq. (35-36), the resistances to chemical fluxes in the electrolyte and electrode are functions of the concentration of present species. As a result, in a volume with a higher concentration the further addition of species is associated with a higher potential than in one with a lower concentration. Local concentration gradients in either domain therefore lead to chemical fluxes through these resistances, leading to temporary diffusion losses.

Overall cell potential: Recalling the formulation of the electrode/electrolyte interface potentials for each electrode (Eq. 4) the overall battery operating voltage $V_{\text {bat }}$ is obtained as the sum of all the potential drops 
across the cell. From the positive to the negative current collector (right to left in Fig. 4), the total voltage drop can be expressed as

$$
\begin{aligned}
V_{\mathrm{bat}}= & -R_{\mathrm{e}^{-}}^{\mathrm{el}, \mathrm{c}} \cdot I_{\mathrm{bat}} \quad \rightarrow \text { Ohmic potential drop at cathode/current collector } \\
& +\left(\phi_{\mathrm{c}}^{\mathrm{s}}-\phi_{\mathrm{c}}^{\mathrm{l}}\right) \quad \rightarrow \text { potential difference at cathode/electrolyte interface } \\
& -\left(\phi_{\mathrm{c}}^{\mathrm{l}}-\phi_{\mathrm{a}}^{\mathrm{l}}\right) \quad \rightarrow \text { potential difference across electrolyte } \\
& -\left(\phi_{\mathrm{a}}^{\mathrm{s}}-\phi_{\mathrm{a}}^{\mathrm{l}}\right) \quad \rightarrow \text { potential difference at anode/electrolyte interface } \\
& -R_{\mathrm{e}^{-}}^{\mathrm{el,a}} \cdot I_{\mathrm{bat}} \quad \rightarrow \text { Ohmic potential drop at anode/current collector }
\end{aligned}
$$

The Ohmic contact resistances $R_{\mathrm{e}^{-}}^{\mathrm{el}}$ encapsulate the electronic Ohmic loss through the cathode aluminium current collector and the anode copper current collector as well as the electronically conducting additives in both electrodes and the contact between the domains.

Information on electrode equilibrium potential and overpotential contributions can be obtained during the running of the model by using a virtual voltmeter, as potential differences and currents in the circuit are direct representations of physical potentials and fluxes inside the battery.

\section{Model implementation and results}

In the following the model's strengths and capabilities are demonstrated through various representative studies.

\subsection{Model Parametrisation}

The phenomenological equivalent circuit model was implemented in Matlab/Simulink (Version 14b) and the simulation results obtained with a variable step Bogacki-Shampine ODE23 solver. Model predictions were compared to experimental data obtained with a commercial $\mathrm{LiNi}_{0.33} \mathrm{Co}_{0.33} \mathrm{Mn}_{0.33} \mathrm{O}_{2}$ graphite pouch 
cell SLPB 11043140H (5 Ah) by Dow Kokam. All studies were performed on the same 'fresh' cell to eliminate the possible effect of manufacturing differences. The cell parameters were obtained from the literature (with the relevant references provided in Table 2) with none being additionally fitted beyond what was done by the original parameter's authors. These parameters have been measured, estimated or fitted by these sources and reflect the real battery characteristics to the best of the authors' knowledge.

The graphite anode thermodynamic potential as a function of stoichiometry was assumed as in $[36,37]$, whereas the equation for the cathode was obtained as the difference of a near-OCV experiment (constant current discharge at $\mathrm{C} / 20$ ) and the anode thermodynamic potential (equations given in Table 2).

\subsection{Model application to various load cycles}

Discharges at 0.5 C, 1 C, 2 C, 5 C, 10 C, 20 C and charges at 0.5 C, 1 C and 2 C were conducted in a thermally controlled environment at room temperature using a potentiostat (HCP/1005 Bio-Logic) with a 100 A booster.

Model predictions and experimental data can be seen in Fig. 5(a) and (b). The simulation results show good agreement with the experimental data, even for the higher discharge rates. The simulated temperature increase encountered during 10 C, 5 C, 2 C and 1 C discharge procedures is shown in Fig. 5(c) with experimental data shown for comparison.

All data traces exhibit non-linear temperature increases which are partly due to non-linear entropic heat contributions as well as to the non-linear increase of charge-transfer resistances with SOC. The heat contributions in each electrode arising during a $2 \mathrm{C}$ discharge are plotted in in Fig. 5(d). 
One of the novel contributions of this model is the observability of any physical parameter during simulation. Only the overall effect thereof is predicted in conventional 'black box' models. In the following the model's capability in unlocking 'hidden' information is demonstrated by analysing the various overpotentials which contribute to the overall cell overpotential. The various overpotential contributions during a full $2 \mathrm{C}$ discharge are shown in in Fig. 6(a). The activation potential dominates nearly throughout, although at very low SOC diffusion overpotentials dominate. The diffusion overpotential contributions are relatively insignificant at high to medium SOC, and become significant at low SOC. This is in line with internal impedance characteristics observed in the Electrochemical Impedance Spectroscopy (EIS) measurement in in Fig. $6(\mathrm{~b})$, where the arc at $100 \%$ SOC is very small compared to that at $0 \%$ SOC. This EIS analysis of the Kokam test cell at various SOCs was carried out to compare the model's predictions with EIS fitted circuit parameters which are usually used in BMS designs. These measurements were performed with a Biologic VSP potentiostat under application of a 2 C DC load superimposed with a $200 \mathrm{~mA}$ RMS AC current sine oscillation.

The resistance contributions due to the various overpotentials in Fig. 6(a) are shown in Fig. 6(c) and Fig. 6(d). In these figures the diffusion and charge-transfer resistances as predicted by fitted EIS data in in Fig. $6(b)$ are compared to the overall diffusion and charge-transfer resistances as predicted by the model.

It can be seen that the new model allows a differentiation of the individual sources of resistances and for the dominant sources singled out: diffusion in the cathode followed by diffusion in the anode. This resulting direct access to individual resistance contributions in the model is necessary for the implementation of degradation phenomena.

The evaluation of exchange current density at anode and cathode separately is important for the further calculation of side reaction current densities leading to the accumulation of lost cyclable material in the passivating layer at either electrode. This allows the prediction of capacity fade as a function of lost active 
material shuttling between anode and cathode. Fig. 7(a) shows a comparison of experimental and simulated capacity fade over 1000 cycles of a 6 C discharge followed by a 2 C charge for a cell with tab temperature maintained at $20^{\circ} \mathrm{C}$ (for details of the experimental setup, see [38]). Cell degradation is further visualised in Fig $7(b)$ where the simulation results are plotted as incremental capacity curves $(\mathrm{dQ} / \mathrm{dV})$ and compared to experimental data. The changes brought on by the loss of cyclable lithium as well as the reduction in cell potential brought on by an increase in internal resistance can be seen. The $\mathrm{dQ} / \mathrm{dV}$ curve fits are not perfect but do show the correct trends particularly for the main peak at $3.7 \mathrm{~V}$, and the errors are most likely due to only one degradation mechanism with two consequences being included in this model: passivating layer growth leading to capacity fade due to loss of cyclable lithium and power fade due to a progressive increase of internal resistance. This is the first version of the model and further modes of degradation are currently being investigated and will be added to the model to improve its accuracy and fit to experimental data.

The simulation results and experimental data of a charge/discharge pulse test procedure pulse of $170 \mathrm{~s}$ duration are shown in Fig. 8(a): a 5 C discharge (20s) is followed by an open-circuit relaxation period (90 s), a 5 C charge (20 s) and an open-circuit relaxation period (30 s). The pulse procedure was applied to a 5 Ah Kokam battery cell at various SOC levels $(80 \%, 65 \%, 50 \%, 35 \%$ and $20 \%$ SOC) with the aim to demonstrate the model's capability in evaluating dynamic load inputs over the whole range of SOCs.

To demonstrate the model's capabilities in a real-world application, test data (current, voltage, time) was collected from a single cell operating as part of a battery pack of a vehicle participating in the Future Car Challenge in 2012 [39]. Of this, a 25 min window, shown in Fig. 8 (b) was used as input to the model and the results compared to the cell voltage recordings made during the run. The vehicle in this case was a PHEV with relatively low C-rates. The cell measurements $(\square)$ were recorded from the BMS via the vehicle CAN (Controller Area Network) bus at discrete time steps (0.1s) with an associated allowable error (I) in Fig. 8. 
Assuming a brand-new cell of this test vehicle under investigation, the error between test data and ECN results is attributed mostly to wire shielding (errors in current/voltage recordings of the test data) and temperature impact, possibly from neighbouring cells (error in simulation results). Those errors (average $2.35 \%-91.65 \mathrm{mV}$ ) were found to be worst during the most aggressive $5 \%$ of discharge events (in terms of C-rate) whilst all remaining events were found to deviate by less than $0.12 \%$ from the collected data. Runtime of the simulation was $42.57 \mathrm{~s}$ for the whole 25 min load-cycle using the same computation power available as previously mentioned.

\section{Conclusion}

The model presented in this section addresses the majority of aspects previously identified as areas of further development

- In the presented methodology the model is structured into clearly defined physics based sub-circuits governing individual species' phenomena. Each can be observed separately and the evolution of all model variables (system states) can be compared to other model software or, where accessible, to experimental data.

- An electrical analogy, the Triple Species Element is derived for the electrochemical reaction in the presence of an electrochemical double layer and passivating layers which links the sub-systems of the individual species. This TSE allows for a straightforward comparison of electrochemical parameters such as charge-transfer resistance to EIS experimental data.

- The newly presented phenomenological equivalent circuit network allows for locally variable

(a) passivating layer thickness, resistance and capacitance, and

(b) double-layer capacitance and potential depending on local electrochemistry and temperature. 
- Previous advanced ECN developments have been extended to include phenomena thus far omitted. These are

(a) local temperature variation and the subsequent dependency of local electrochemical processes,

(b) capacity fade through loss of cyclable lithium, and

(c) power fade through increase of internal impedance

- In this model implementation the dependency on an empirical relation governing the equilibrium potential of each electrode (due to its standard reduction potential and concentration variation) has not been removed. The simplicity of the experiments required in order to extract these two functions rendered this method superior for the purpose of automotive applications compared to the detailed half-cell testing and atomic physics analysis that would be required for a fundamentally derived function.

- Previous work has been extended upon in terms of visual representation and layout of the network. This allows for straightforward future adaptations, modifications, extension and system-level implementation by system designers.

In comparison to previous ECN modelling efforts found in the literature, the presented phenomenological equivalent circuit network allows the extraction of a wealth of additional information on internal battery performance characteristics (e.g. concentrations, potentials and overpotentials), enabling a more accurate real-time prediction of the states of the battery. Given the same measurement inputs as conventional 'black-box' models implemented in commercial vehicles today, this information allows the quantification of battery performance parameters (such as local heat generation and capacity/power fade), which have so far been inaccessible. The proposed model therefore allows a significant enhancement of BMS capabilities while avoiding costly hardware modifications. This will not only enable designers to better estimate the lifetime of the product as a whole for warranty purposes, but will also render electric vehicles a compelling alternative to internal combustion vehicles. 


\section{Acknowledgements}

The authors would like to thank Yu Merla, Yan Zhao, Mohd Ibthisham Ardani and lan Hunt for collecting experimental data. The authors would also like to acknowledge Climate KIC for the PhD funding of MarieTherese von Srbik and the EPSRC for funding of this work through a Career Acceleration Fellowship for Gregory Offer, award number EP/I00422X/1.

6. $\quad$ References

[1] D. Andre, M. Meiler, K. Steiner, H. Walz, T. Soczka-Guth, D.U. Sauer, J. Power Sources 196 (2011) 5349.

[2] D. Andre, M. Meiler, K. Steiner, C. Wimmer, T. Soczka-Guth, D.U. Sauer, J. Power Sources 196 (2011) 5334.

[3] S. Buller, The Attached Dissertation Work Contents, 2003.

[4] S. Buller, M. Thele, R.W.A.A. DeDoncker, E. Karden, IEEE Trans. Ind. Appl. 41 (2005) 742.

[5] S. Buller, M. Thele, E. Karden, R.W. De Doncker, J. Power Sources 113 (2003) 422.

[6] S. Lee, J. Kim, J. Lee, B.H. Cho, J. Power Sources 185 (2008) 1367.

[7] V. Pop, H.J. Bergveld, D. Danilov, P.P.L. Regtien, P.H.L. Notten, (2008).

[8] J.P. Schmidt, H.Y. Tran, J. Richter, E. Ivers-Tiffée, M. Wohlfahrt-Mehrens, J. Power Sources 239 (2013) 696.

[9] O. Tremblay, L.A. Dessaint, A.I. Dekkiche, in:, 2007 IEEE Veh. Power Propuls. Conf., IEEE, 2007, pp. 284-289.

[10] M. Dorri, a. H. Shamekhi, Proc. Inst. Mech. Eng. Part D J. Automob. Eng. 225 (2011) 747.

[11] H. He, X. Zhang, R. Xiong, Y. Xu, H. Guo, Energy 39 (2012) 310.

[12] H.J. Bergveld, Battery Management Systems Design by Modelling, University of Eindhoven, 2001. 
[13] S. Raël, M. Hinaje, J. Power Sources 222 (2013) 112.

[14] A.J. Bard, L.R. Faulkner, Electrochemical Methods - Fundamentals and Applications, 2nd ed., Wiley \& Sons, New York, New York, USA, 2001.

[15] J.A. V. Butler, Trans. Faraday Soc. 19 (1924) 729.

[16] N. Chaturvedi, R. Klein, J. Christensen, J. Ahmed, A. Kojic, IEEE Control Syst. Mag. 30 (2010) 49.

[17] K. Smith, C.Y. Wang, J. Power Sources 160 (2006) 662.

[18] K.A. Smith, C.D. Rahn, C.Y. Wang, in:, 2008 IEEE Int. Conf. Control Appl., IEEE, 2008, pp. 714-719.

[19] M. Safari, M. Morcrette, A. Teyssot, C. Delacourt, J. Electrochem. Soc. 156 (2009) A145.

[20] M. Safari, C. Delacourt, J. Electrochem. Soc. 158 (2011) A63.

[21] H.J. Bergveld, P.H.L. Notten, V. Pop, Apparatus and Method for Determination of the State-ofCharge of a Battery When the Battery Is Not in Equilibrium, 2010.

[22] R.E. White, J.O. Bockris, B.E. Conway, Modern Aspects of Electrochemistry, Springer US, Boston, MA, 1983.

[23] E. Prada, D. Di Domenico, Y. Creff, J. Bernard, V. Sauvant-Moynot, F. Huet, J. Electrochem. Soc. 159 (2012) A1508.

[24] E. Prada, D. Di Domenico, Y. Creff, J. Bernard, V. Sauvant-Moynot, F. Huet, J. Electrochem. Soc. 160 (2013) A616.

[25] G. Ning, B.N. Popov, J. Electrochem. Soc. 151 (2004) A1584.

[26] K.B. Oldham, J. Electroanal. Chem. 613 (2008) 131.

[27] A.A. Kornyshev, J. Phys. Chem. B 111 (2007) 5545.

[28] J.C. Forman, S.J. Moura, J.L. Stein, H.K. Fathy, J. Power Sources 210 (2012) 263.

[29] T.F. Fuller, M. Doyle, J. Newman, J. Electrochem. Soc. 141 (1994) 1.

[30] M. Doyle, J. Electrochem. Soc. 143 (1996) 1890.

[31] M. Doyle, J.P. Meyers, J. Newman, J. Electrochem. Soc. 147 (2000) 99.

[32] T.F. Fuller, J. Electrochem. Soc. 141 (1994) 1.

[33] J. Newman, K.E. Thomas-Alyea, Electrochemical Systems, 3rd ed., Wiley \& Sons, New York; USA, 2004.

[34] A. Fick, Ann. Der Phys. Und Chemie 170 (1855) 59.

[35] J. Horno, M.T. García-Hernández, C.F. González-Fernández, J. Electroanal. Chem. 352 (1993) 83. 
[36] K. Smith, C.Y. Wang, J. Power Sources 160 (2006) 662.

[37] K. Smith, C.Y. Wang, J. Power Sources 161 (2006) 628.

[38] Y. Troxler, B. Wu, M. Marinescu, V. Yufit, Y. Patel, A.J. Marquis, N.P. Brandon, G.J. Offer, J. Power Sources 247 (2014) 1018.

[39] M.-T. von Srbik, B. Cussons, R.F. Martinez-Botas, L. Lytton, in:, Hybrid Electr. Veh. Conf. 2013 (HEVC 2013), Institution of Engineering and Technology, 2013, pp. 8.1-8.1. 


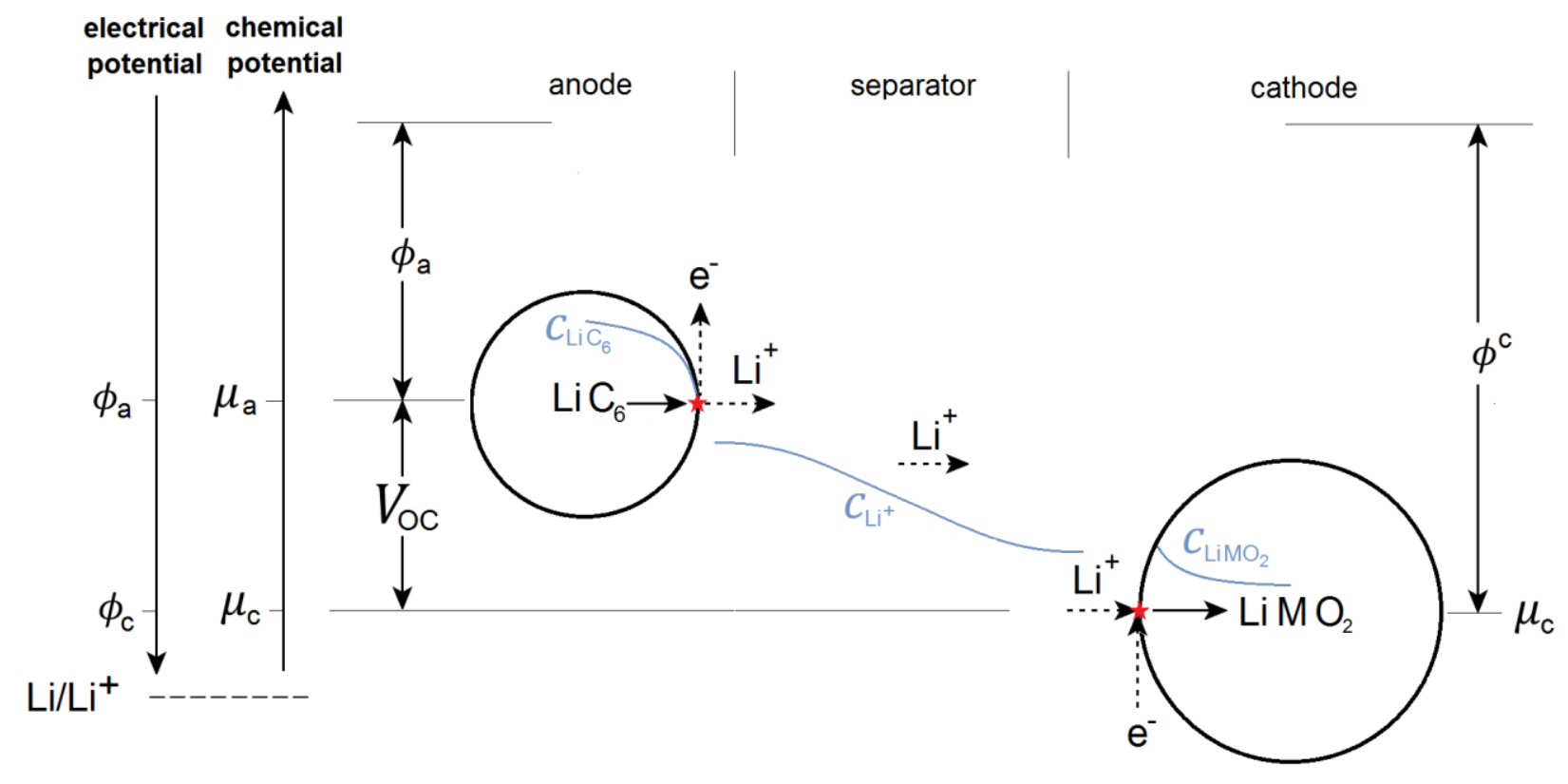




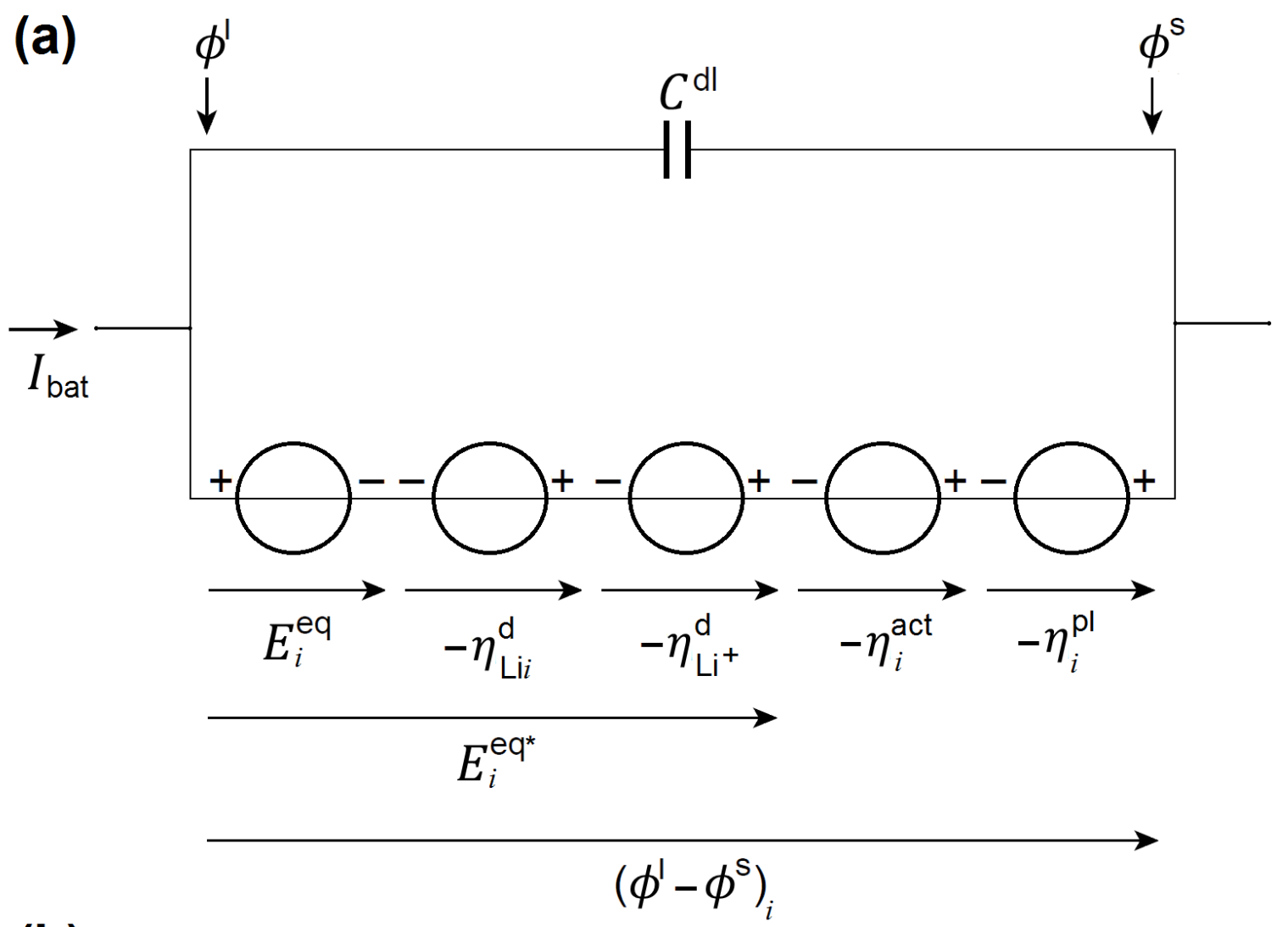

(b)

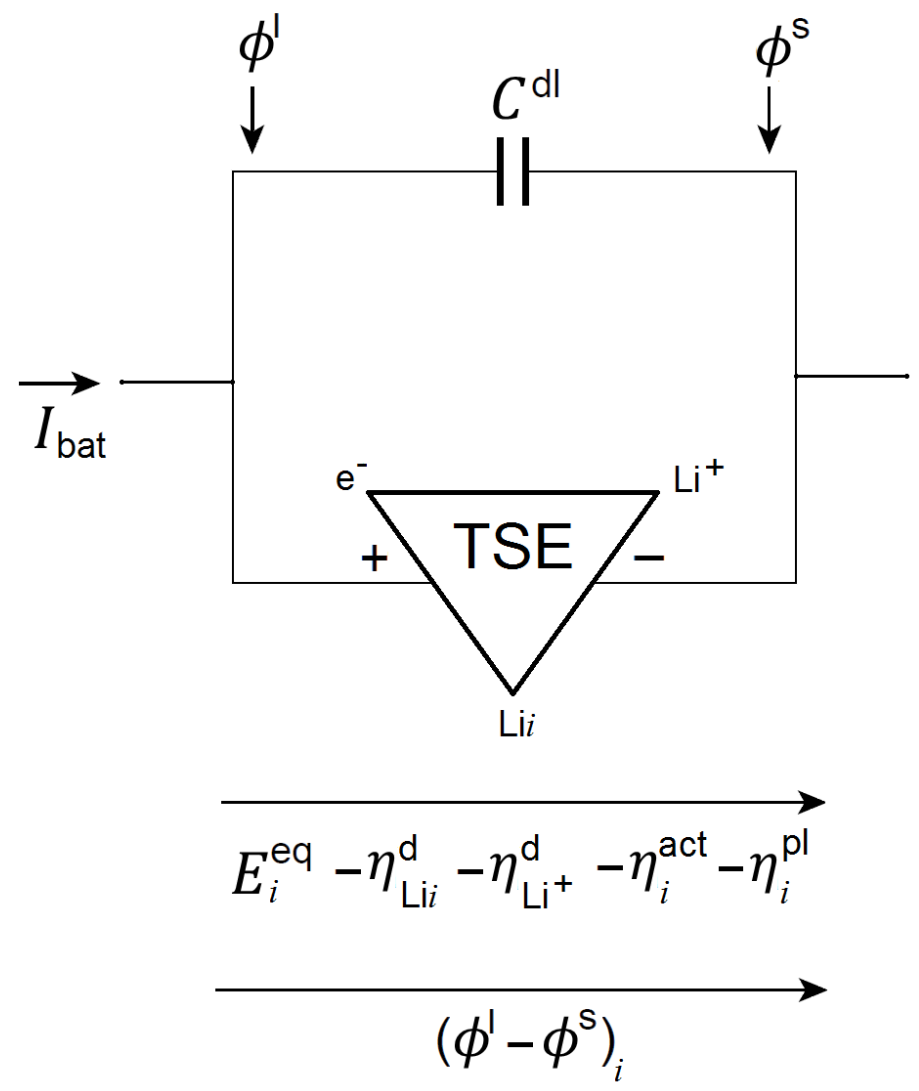



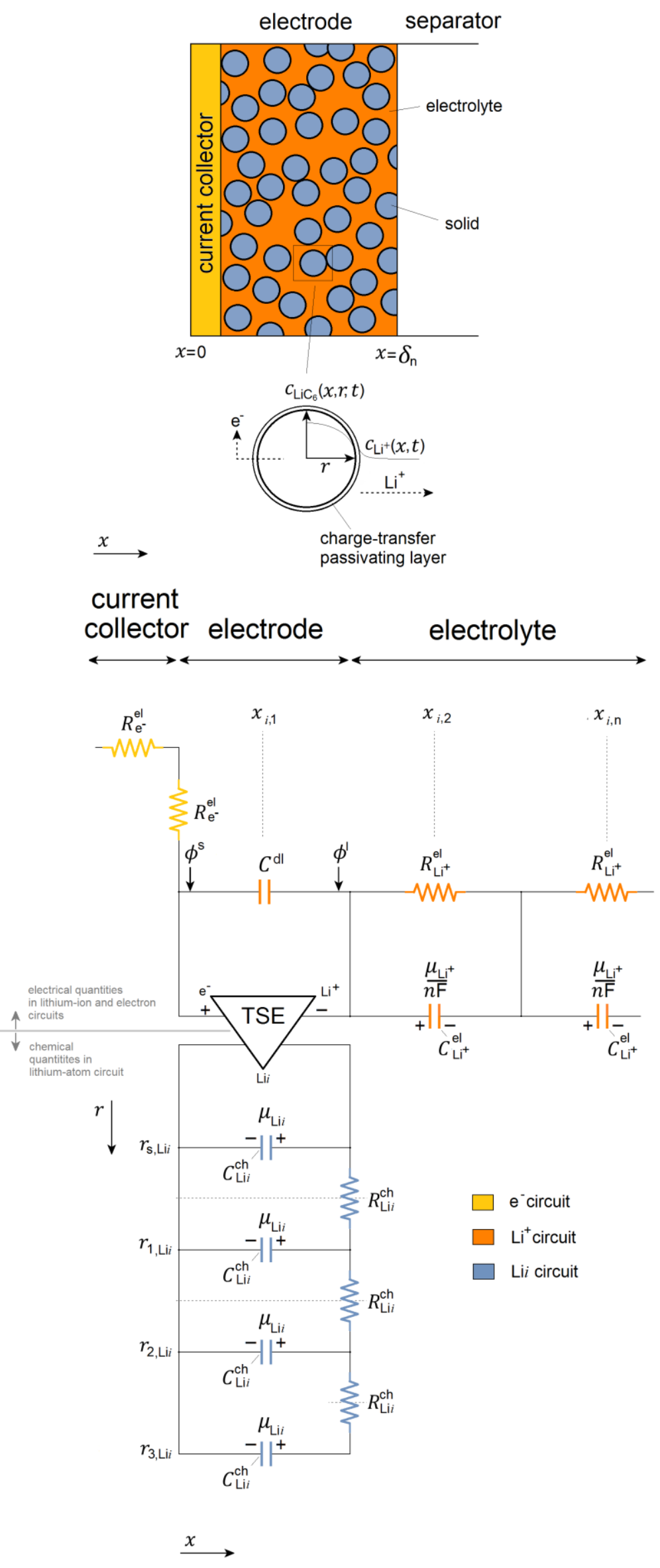


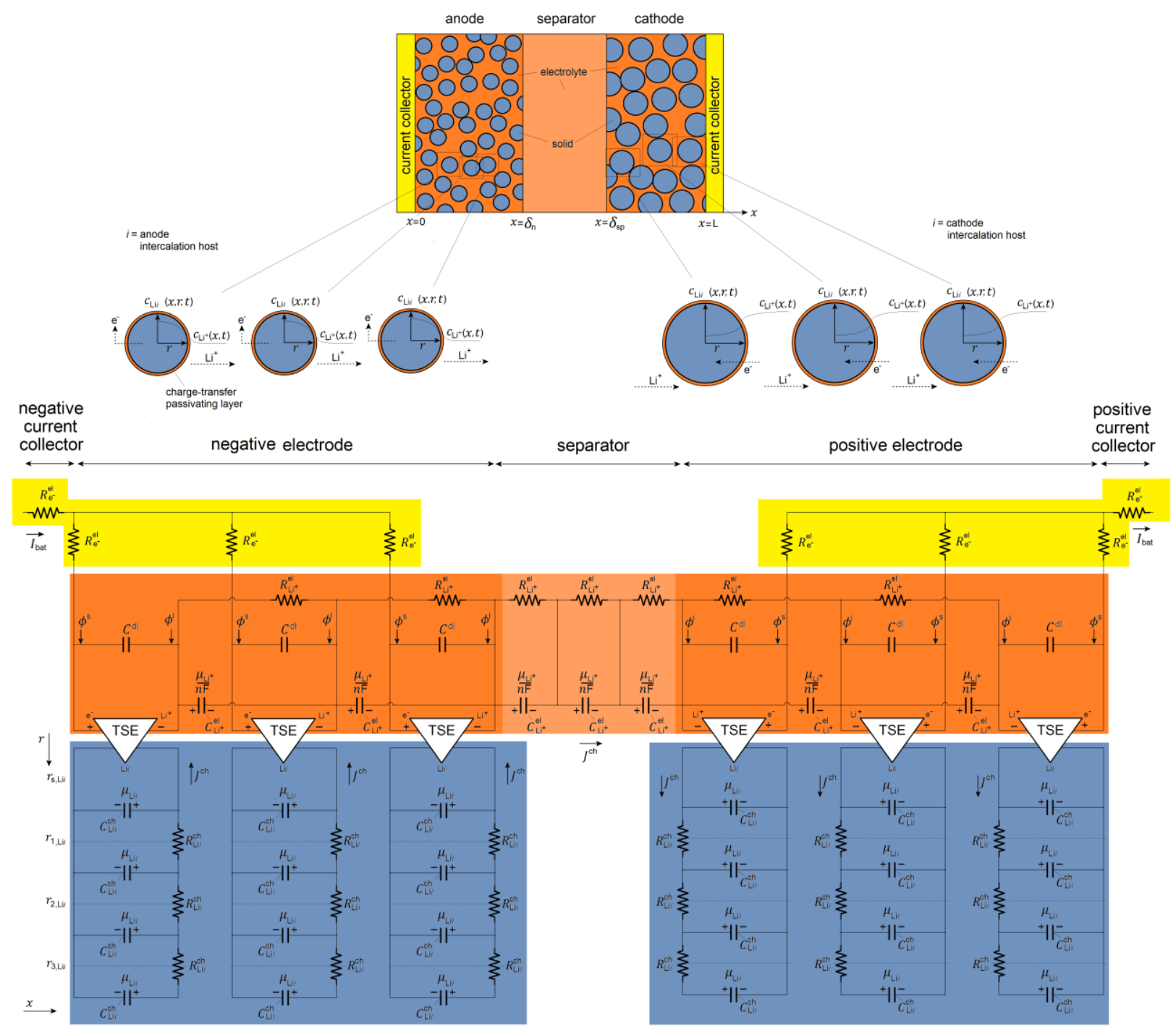


(a)

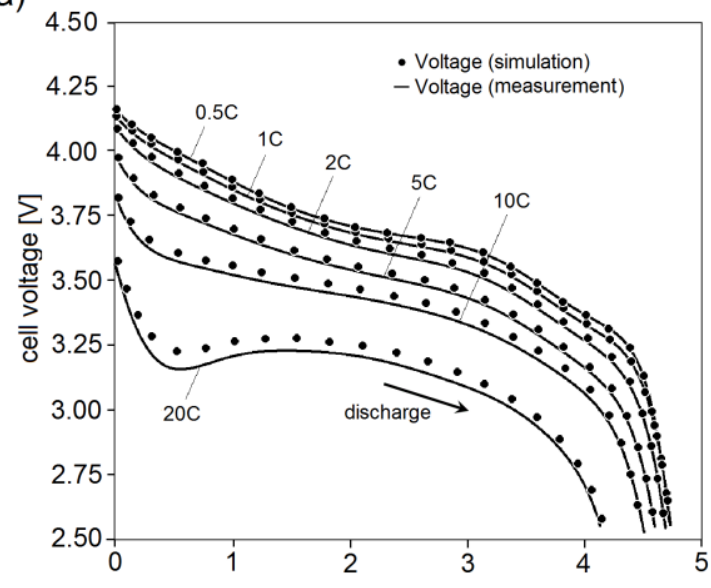

(c)

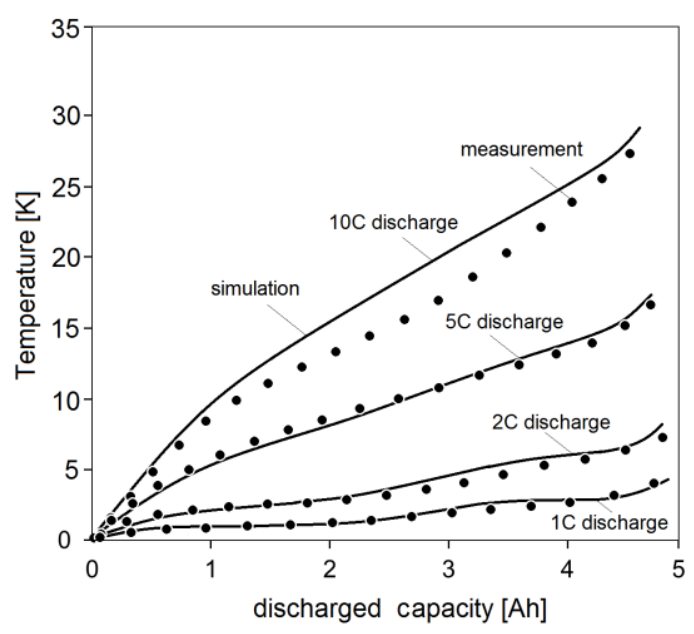

(b)
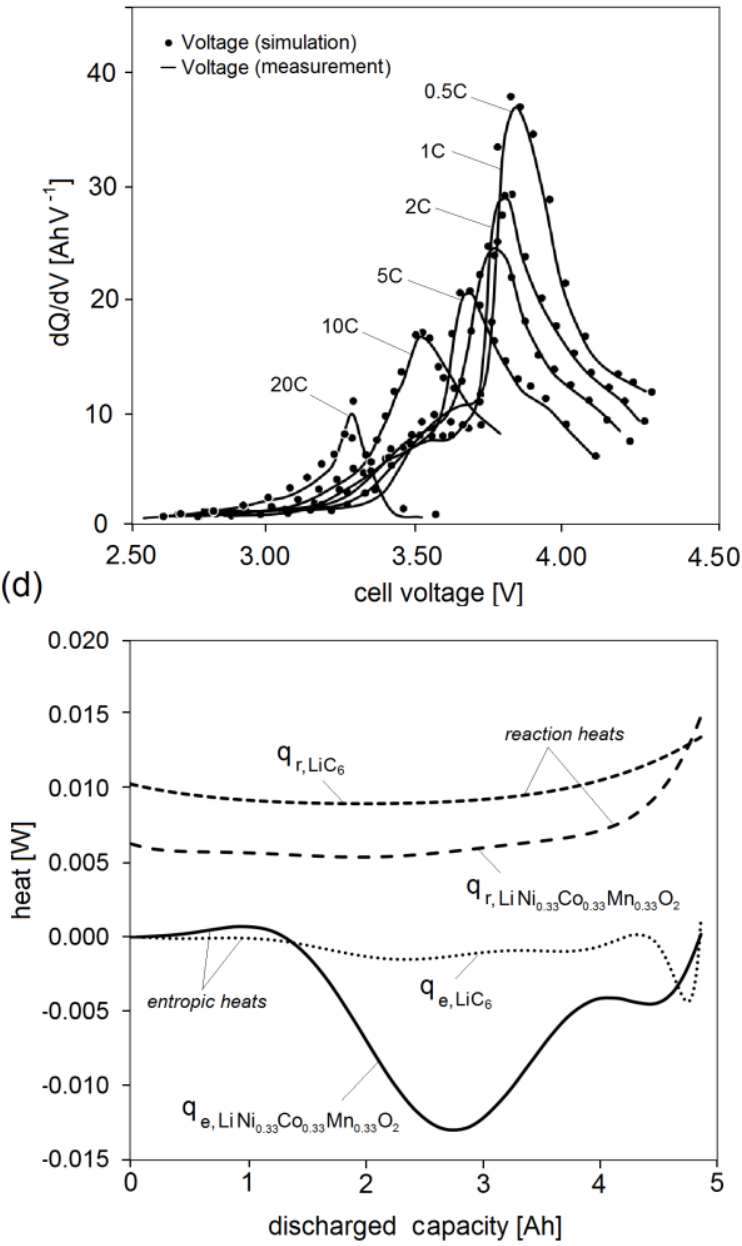
(a)

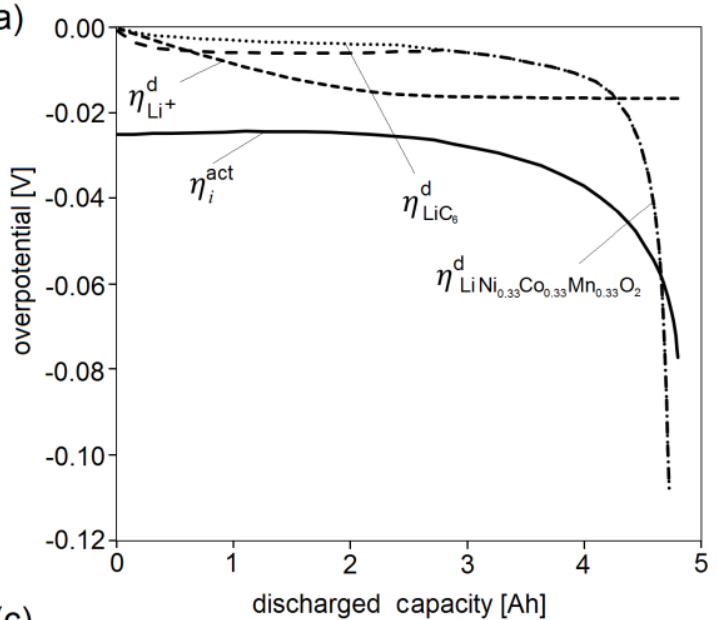

(c)

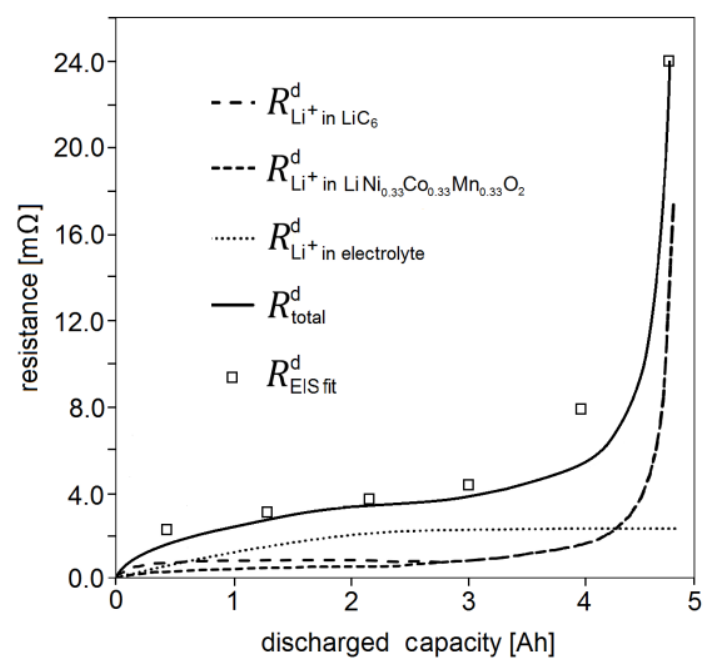

(b)

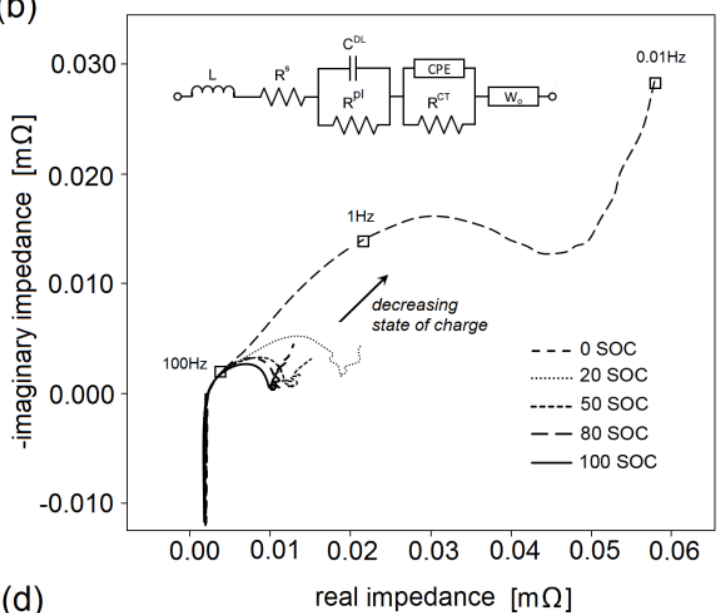

(d)

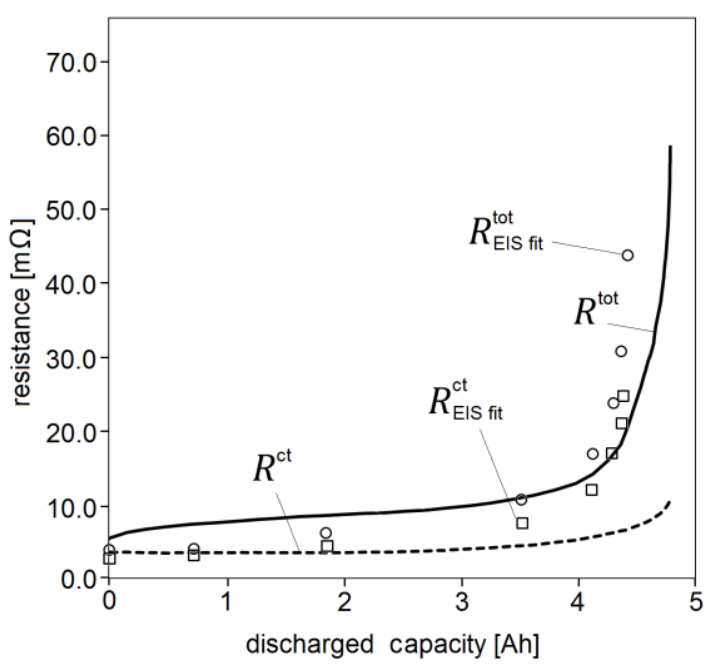


(a)

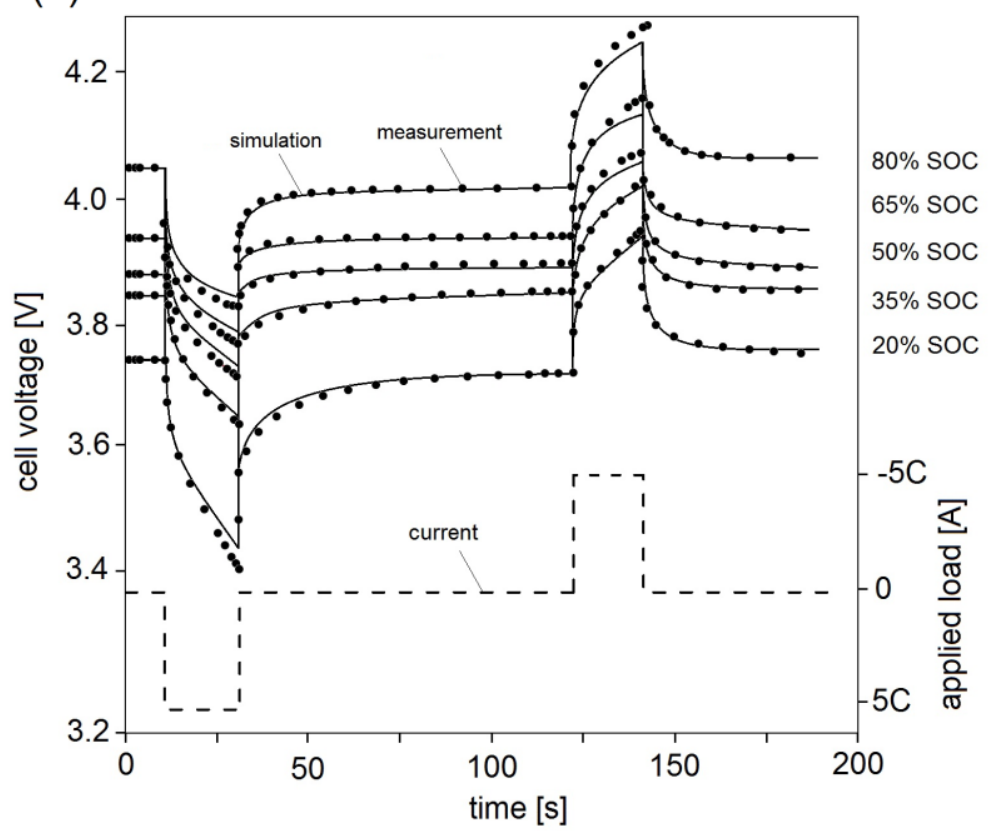

(b)

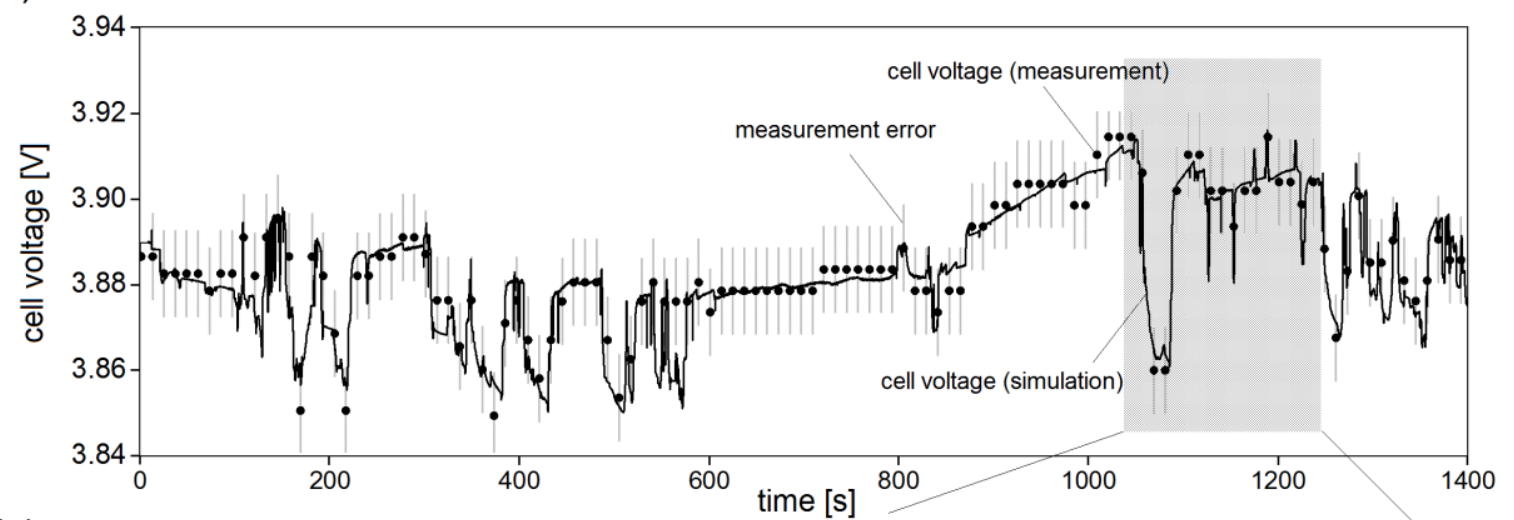

(c)

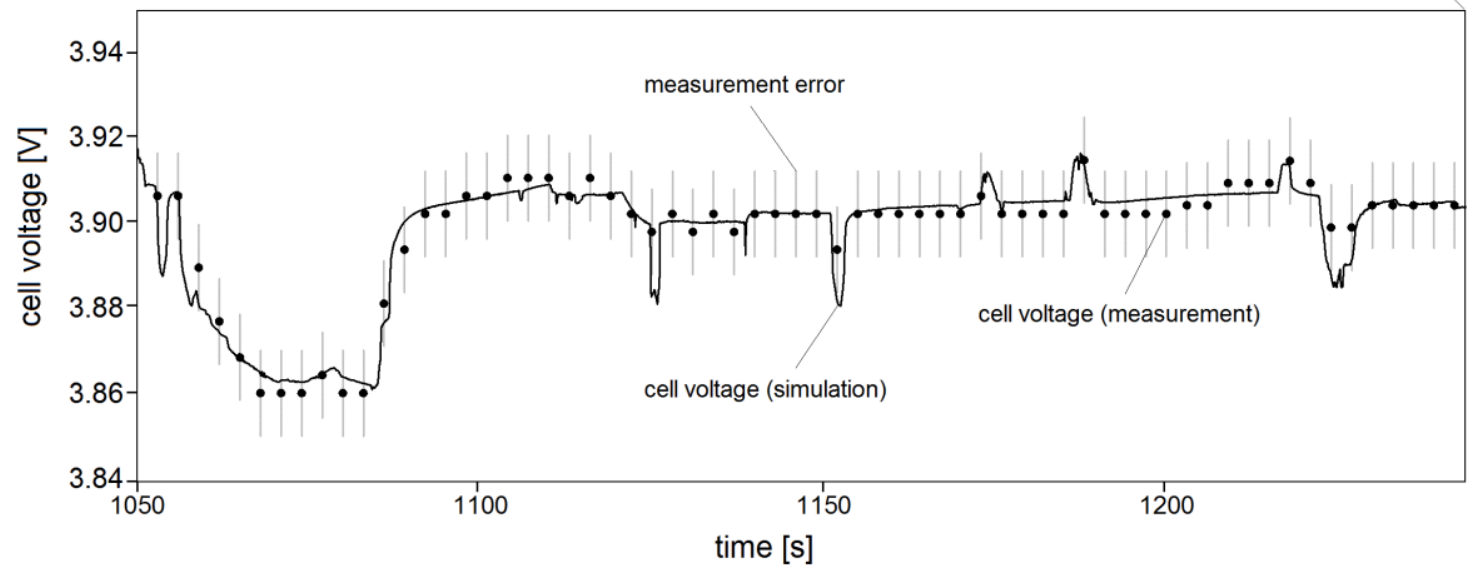


(a)

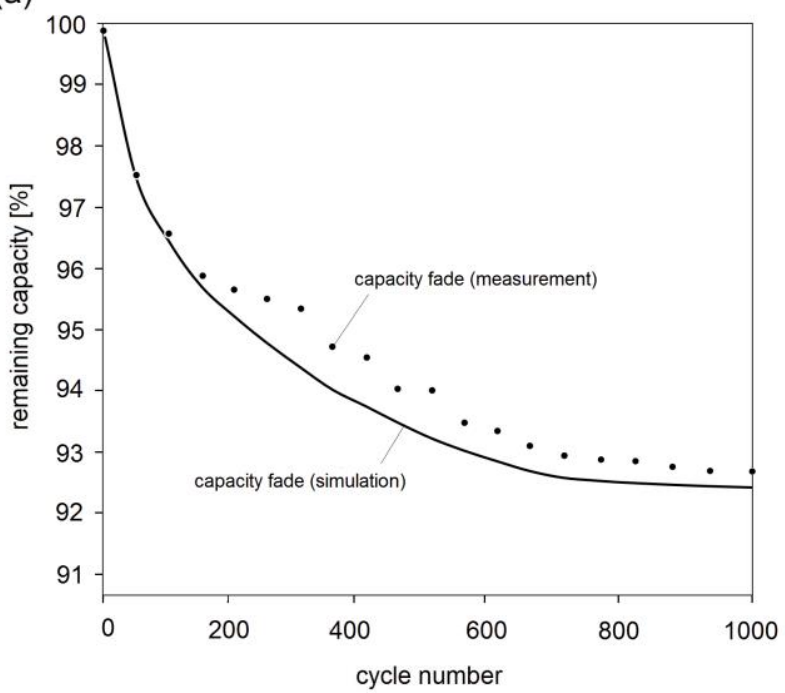

(b)

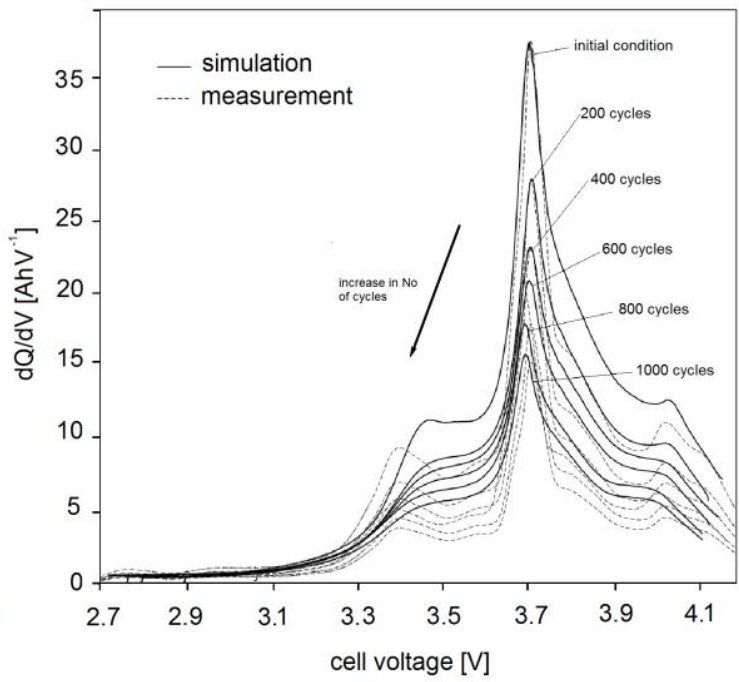

